\title{
Soft food microrheology
}

\author{
Jiakai Lu*, Carlos M. Corvalan* \\ Transport Phenomena Laboratory, Department of Food Science, Purdue University, West \\ Lafayette, IN 47907, USA
}

\begin{abstract}
Soft food materials, such as gels, pastes, emulsions and colloidal suspensions, are highly heterogeneous structures under constant dynamic interactions. For this reason, these materials frequently exhibit complicated rheological responses, many of which defy characterization by conventional techniques without disrupting their internal food microstructure. In this paper, we highlight recent advances in microrheological methods to probe rheological responses of delicate samples at the microscale. They offer a powerful new approach to characterize soft food materials in a direct and unobstrusive way. Microrheological methods promise to reveal a better fundamental understanding of the underlying structures and dynamics of soft food materials, inform novel constitutive models, and help develop new technological applications.
\end{abstract}

Keywords: food, rheology, microrheology

\footnotetext{
* Corresponding author.

Email address: 1u3@purdue.edu (Jiakai Lu)
} 


\section{INTRODUCTION}

Food rheology is the study of how food materials deform in response to mechanical stresses. Food materials - and especially soft food materialsare typically composed of heterogeneous microstructures and metastable arrangements that lead to complicated responses when subjected to stress (Fischer and Windhab, 2011). Moreover, experimental techniques able to quantify the rheology of food materials at small length scales can provide basic insight into their underlying structure and dynamics, and ultimately into their response during processing, storage and consumption.

Microrheological techniques have been developed to complement and extend conventional bulk rheology by measuring local rheological properties of a material at the micron scale. In these techniques, the rheological information is extracted from the motion of microscopic probe particles embedded within the material. The embedded probe particles are driven by random thermal fluctuations in passive microrheology (**Squires and Mason, 2010), or by an applied external force in active microrheology (Wilson and Poon, 2011). Both active and passive microrheology are increasingly been used to characterize challenging natural and synthetic materials, but these methods have not yet been fully exploited in food rheology. Here, we briefly discuss recent progress in microrheology, and highlight benefits of these technique for studies of soft materials that play a critical role in food technology such as gels, colloids and biopolymers. 


\section{ACTIVE AND PASSIVE MICRORHEOLOGY}

Microrheology describes a wide collection of techniques used to quantify phenomena involved in the storage and dissipation of mechanical energy in a microscopic region of a material. In the last two decades there have been important developments in microrheological techniques, which can be broadly classified as passive or active depending on the forces underlying the measurements.

Passive microrheology quantifies the mechanical properties of a sample by relating the diffusive fluctuations of an embedded probe particle to the shear modulus of the material (Fig. 1). This technique is ideally suited for soft food materials because the weak thermal energy $\left(\sim \kappa_{B} T\right)$ required to fluctuate the particles provides a delicate probe of the internal food microstructure. And because there is no need to apply an external force, it only requires relatively simple instrumentation to trace the motion of the particles such as direct visualization (Vadas et al., 1973; Filipe et al., 2010), laser deflection tracking (Mason and Weitz, 1995; Du et al., 2012), diffusing wave spectroscopy (Mason et al., 1997; Oelschlaeger et al., 2013) or dynamic light scattering (Mason et al., 1996).

The formalism to extract rheological information from thermal fluctuations employs the generalized Stokes-Einstein relation. In the simplest case of a purely viscous material, the mean-square displacement of the fluctuating particles $\left\langle\Delta r^{2}\right\rangle=6 D t$ depends linearly on time through the diffusion coefficient $D$. For a probe particle of (hydraulic) radius $a$, the diffusion coefficient is given by the Stokes-Einstein equation $D=\kappa_{B} T /(6 \pi \eta a)$, which relates the desired sample viscosity $\eta$ to the measured mean-square displacement 
(**Squires and Mason, 2010).

In a seminal work for modern microrheology, Mason and Weitz (1995) demonstrated that this approach could be generalized to characterize soft materials that exhibit viscoelastic behavior using the fluctuation-dissipation theorem (Landau and Lifshitz, 2000; Evans et al., 2016). By employing the fluctuation-dissipation theorem, Mason and Weitz (1995) generalized the Stokes-Einstein equation to relate the measured mean-squared displacement $\left\langle\Delta r^{2}\right\rangle$ of the particles (Figure 1a) directly to the sample frequency-dependent viscosity $\eta^{*}(\omega)$, and therefore to the sample complex shear modulus $G^{*}(\omega)=$ $i \omega \eta^{*}(\omega)=G^{\prime}(\omega)+i G^{\prime \prime}(\omega)$ via integral transforms (Figure 1b). For a detailed discussion of the application of the generalized Stokes-Einstein relation to passive microrheology and conditions that can invalidate this generalization, see **Squires and Mason (2010); DePuit and Squires (2012a,b).

Active microrheology, instead, extract the mechanical properties of the material from the motion of embedded microscopic particles driven by an external force (Wilson and Poon, 2011). Therefore, besides instruments to trace the motion of the particles, active microrheology requires additional equipment to actively drive them within the material. Generally, the probe particles are pulled by a constant force using magnetic tweezers (Habdas et al., 2004) or they are driven at constant speed using optical tweezers (Furst, 2003; Sriram et al., 2010; Meyer et al., 2006). However, unlike passive techniques, active microrheology enable the characterization of the non-linear rheology of materials by using the applied force to perturb their microstructure far away from equilibrium (Zia and Brady, 2013; Gomez-Solano and Bechinger, 2014; *Gomez-Solano and Bechinger, 2015). 
Currently, a frontier research area in microrheology involves combining the benefits of both active and passive approaches. In active microrheology, probe particles are driven by external forces, whereas in passive microrheology the particles are driven by Brownian fluctuations. Combined methods involve the motion of an embedded probe that is actively pulled by internal but non-Brownian stresses (e.g. by capillarity). Recently, rigorous theories elucidating the interplay between endogenous capillary forces and the hydrodynamics of embedded microscopic pores and indentations have been developed (McGraw et al., 2011; Rahmani et al., 2014; *Backholm et al., 2014; Lu and Corvalan, 2015; * Lu et al., 2015), and applied to relate the motion of the probe "micropores" (Fig. 2a) to the Newtonian (Fig. 2b, black line), shear-thinning (Fig. 2b, blue line), and viscoelastic rheology of the material (Roth and Dutcher, 2006; McGraw et al., 2011).

\section{APPLICATION TO FOODS}

Microrheology is particularly attractive for the study of soft materials such as gels, colloids, and biopolymers that play an ubiquitous role in food science and food technology. Because of the small size of the embedded probe particles, it can measure local rheological properties that are necessarily lumped in macroscopic measurements (Schultz and Furst, 2012). And because of the low inertia of the particles, it can extract this information at a higher range of frequencies than allowed by conventional rheology (Willenbacher and Oelschlaeger, 2007).

Consequently, an important research area open to food microrheology concerns heterogeneous materials where the rheology changes from point to 
point. Either by successively tracking single probe particles or by simultaneously tracking multiple probe particles at various places in the sample, microrheology can provide insight into the spatial distribution of the internal microstructure for heterogeneous food systems — from dilute suspensions to arrested systems such as gels and colloidal glasses.

Early works in this area include suspensions of wheat gliadin (Xu et al., 2002), a protein responsible for dough viscoelasticity, and suspensions of oat beta-glucan, a bioactive component credited with health benefits (Xu et al., 2007). More recently, studies on suspensions of barley beta-glucan corroborated that microrheology can probe micro-heterogeneities in food samples that go unnoticed by macroscopic rheology (Moschakis et al., 2012).

Since the pioneering work by Mason and Weitz (1995) on hard-sphere colloidal suspensions, the microrheology of colloidal systems has been extensively studied in both the dilute limit and the arrested limit close to the glass transition. In the dilute limit, colloidal suspensions have been studied using microrheological models based on the two-body solution of the Smoluchowski equation as an approximation to the many-body interactions between colloidal particles (Zia and Brady, 2010, 2013). In this limit, microrheology enabled accurate characterization of both the loss modulus $G^{\prime \prime}$, which generally dominates the dynamics, and the growth of the storage modulus $G^{\prime}$ with the volume fraction (**Puertas and Voigtmann, 2014).

Increasing the volume fraction of the colloidal particles may lead to dynamical arrest, and the suspensions may undergo a glass transition (**Puertas and Voigtmann, 2014). Close to their glass transition, dense colloidal suspensions have been studied using active and passive microrheological models 
based on the Mode-Coupling theory because many-body hydrodynamic interactions generally dominate the mechanical response (Gazuz et al., 2009; Gazuz and Fuchs, 2013; Voigtmann and Fuchs, 2013). In these conditions, colloidal suspensions frequently exhibit rheological behavior similar to other soft glassy materials of interest in food technology because of their shared metastable internal disorder. Measurements largely agree on a predominantly elastic behavior with a storage modulus $G^{\prime}(\omega)$ scaling as a very weak power law of frequency $\omega$, and on the presence of a yield stress above which the material readily flows as a Herschel-Bulkley fluid (Anderson et al., 2013). For a review of recent work on the microrheology of colloidal systems, see **Puertas and Voigtmann (2014).

Microrheology enable not only simultaneous rheological measurements at different locations in a sample but also continuous measurements over time (Larsen and Furst, 2008). This makes this technique highly valuable for the study of food materials with evolving dynamic microstructures. Of particular importance for food technology is the use of microrheology to monitor the dynamics of aging (Rich et al., 2011; Gomez-Solano et al., 2012; Zia et al., 2014; Peng and McKenna, 2016) and the dynamics of gel formation (Narita and Indei, 2016).

Early research in food gels include the gelation dynamics of pectin (Vincent et al., 2007), fibrillar proteins (Corrigan and Donald, 2009), and milk gels (Cucheval et al., 2009). And more recently, the gelation dynamics of betalactoglobulin (Balakrishnan et al., 2010), dairy proteins (Moschakis et al., 2010), and beta-glucan solutions (Moschakis et al., 2012). For a recent review of the microrheology of food gels, see Moschakis (2013). 
Recent advances have enabled the rapid characterization of fast-forming gels, and further improved the ability of microrheology to characterize gelation reactions without disturbing the dynamics and assembly of the incipient network structure. Research by Gomez-Solano et al. (2013) demonstrated the utility of microrheology to follow fast process associated to the rapid propagation of the gelation front into the sol phase during sol-gel transition, and Forde and collaborators (Shayegan and Forde, 2013; Shayegan et al., 2014) used microrheology to elucidate delicate mechanisms of self-assembly by following temporal changes in the shear modulus of gels of collagen fibrils since the very early phases of fibrillar growth .

\section{CONCLUSION}

In this paper, we presented a few examples that provide a glimpse of what is now possible using microrheology, and the unique benefits this technique can offer to food scientists beyond those provided by conventional bulk rheology.

Potential applications of microrheology in food technology are by no means limited to these few examples. In addition to the clear advantage of microrheology to characterize minute amounts of sample (e.g. cells, proteins) (Wirtz, 2009; Josephson et al., 2016), other potential applications include the use of microrheology to interrogate the mechanical properties of bacterial biofilms (Rogers et al., 2008; Galy et al., 2012), and the viscoelastic properties of interfaces and emulsions stabilizers (Mendoza et al., 2014; Huang et al., 2015). Microrheology has also been applied to characterize dynamical processes that modulate the release of the active ingredients in materials used 
for controlled release applications (Frith et al., 2015). Moreover, leading research areas may soon enable automated microrheology instrumentation for high-throughput screening of food materials (*Shindel and Furst, 2015), as well as simpler and less expensive ways to characterize non-linear food rheology by extending microrheology to different driving modes using micropores and indentations as embedded micro and nanorheological probes (Rahmani et al., 2014; Lu and Corvalan, 2016).

Clearly, food microrheology is still in its infancy and there is much interdisciplinary work to be done to further familiarize food scientists with this technique. But the possibilities for deciphering the mechanical response of food materials at an unprecedented level of detail, and using this information to develop better theoretical constitutive models (Brader, 2010) and new technological applications, are enormous.

\section{Acknowledgments}

This work was partially supported by the USDA National Institute of Food and Agriculture, Hatch projects 1008409 and 199888. 


\section{References}

Anderson, D., D. Schaar, H. Hentschel, J. Hay, P. Habdas, and E. R. Weeks (2013). Local elastic response measured near the colloidal glass transition. The Journal of chemical physics 138(12), 12A520.

*Backholm, M., M. Benzaquen, T. Salez, E. Raphaël, and K. Dalnoki-Veress (2014). Capillary levelling of a cylindrical hole in a viscous film. Soft matter 10(15), 2550-2558.

Extending the work by McGraw et al., 2011, this paper provides the required theoretical background, as well as experimental validation, for the application of microindentations to characterize local rheological properties.

Balakrishnan, G., D. Durand, and T. Nicolai (2010). Particle diffusion in globular protein gels in relation to the gel structure. Biomacromolecules 12(2), 450-456.

Brader, J. M. (2010). Nonlinear rheology of colloidal dispersions. Journal of Physics: Condensed Matter 22(36), 363101.

Corrigan, A. M. and A. M. Donald (2009). Passive microrheology of solventinduced fibrillar protein networks. Langmuir 25(15), 8599-8605.

Cucheval, A. S., R. R. Vincent, Y. Hemar, D. Otter, and M. A. Williams (2009). Multiple particle tracking investigations of acid milk gels using tracer particles with designed surface chemistries and comparison with diffusing wave spectroscopy studies. Langmuir 25(19), 11827-11834. 
DePuit, R. J. and T. M. Squires (2012a). Micro-macro-discrepancies in nonlinear microrheology: I. quantifying mechanisms in a suspension of brownian ellipsoids. Journal of Physics: Condensed Matter 24(46), 464106.

DePuit, R. J. and T. M. Squires (2012b). Micro-macro discrepancies in nonlinear microrheology: Ii. effect of probe shape. Journal of Physics: Condensed Matter 24(46), 464107.

Du, K., J. A. Liddle, and A. J. Berglund (2012). Three-dimensional realtime tracking of nanoparticles at an oil-water interface. Langmuir 28(25), 9181-9188.

Evans, D. J., D. J. Searles, and S. R. Williams (2016). Fundamentals of Classical Statistical Thermodynamics: Dissipation, Relaxation and Fluctuation Theorems. John Wiley \& Sons.

Filipe, V., A. Hawe, and W. Jiskoot (2010). Critical evaluation of nanoparticle tracking analysis (nta) by nanosight for the measurement of nanoparticles and protein aggregates. Pharmaceutical research 27(5), 796-810.

Fischer, P. and E. J. Windhab (2011). Rheology of food materials. Current Opinion in Colloid \& Interface Science 16(1), 36-40.

Frith, W., A. Donald, D. Adams, and A. Aufderhorst-Roberts (2015). Gels formed from amino-acid derivatives, their novel rheology as probed by bulk and particle tracking rheological methods. Journal of Non-Newtonian Fluid Mechanics 222, 104-111.

Furst, E. M. (2003). Interactions, structure, and microscopic response: complex fluid rheology using laser tweezers. Soft Materials 1(2), 167-185. 
Galy, O., P. Latour-Lambert, K. Zrelli, J.-M. Ghigo, C. Beloin, and N. Henry (2012). Mapping of bacterial biofilm local mechanics by magnetic microparticle actuation. Biophysical journal 103(6), 1400-1408.

Gazuz, I. and M. Fuchs (2013). Nonlinear microrheology of dense colloidal suspensions: A mode-coupling theory. Physical Review E 87(3), 032304.

Gazuz, I., A. M. Puertas, T. Voigtmann, and M. Fuchs (2009). Active and nonlinear microrheology in dense colloidal suspensions. Physical review letters 102(24), 248302.

Gomez-Solano, J., V. Blickle, and C. Bechinger (2013). Nucleation and growth of thermoreversible polymer gels. Physical Review E 87(1), 012308.

Gomez-Solano, J. R. and C. Bechinger (2014). Probing linear and nonlinear microrheology of viscoelastic fluids. EPL (Europhysics Letters) 108(5), 54008.

*Gomez-Solano, J. R. and C. Bechinger (2015). Transient dynamics of a colloidal particle driven through a viscoelastic fluid. New Journal of Physics 17(10), 103032.

This work provides a novel method to measure transient microrheology of viscoelastic fluids by following an actively dragged colloidal particle with an optical trap.

Gomez-Solano, J. R., A. Petrosyan, and S. Ciliberto (2012). Fluctuations, linear response and heat flux of an aging system. EPL (Europhysics Letters) $98(1), 10007$. 
Habdas, P., D. Schaar, A. C. Levitt, and E. R. Weeks (2004). Forced motion of a probe particle near the colloidal glass transition. EPL (Europhysics Letters) 67(3), 477.

Huang, S., K. Gawlitza, R. von Klitzing, L. Gilson, J. Nowak, S. Odenbach, W. Steffen, and G. K. Auernhammer (2015). Microgels at the water/oil interface: In-situ observation of structural ageing and two-dimensional magnetic bead microrheology. Langmuir.

Josephson, L. L., E. M. Furst, and W. J. Galush (2016). Particle tracking microrheology of protein solutions. Journal of Rheology (1978-present) 60(4), $531-540$.

Landau, L. D. and E. Lifshitz (2000). Statistical physics. Oxford: Butterworth-Heinemann 1980.

Larsen, T. H. and E. M. Furst (2008). Microrheology of the liquid-solid transition during gelation. Physical review letters 100(14), 146001.

Lu, J. and C. M. Corvalan (2015). Free-surface dynamics of small pores. Chemical Engineering Science 132, 93-98.

Lu, J. and C. M. Corvalan (2016). Scaling laws for the contraction of nonnewtonian pores. In preparation.

${ }^{*} \mathrm{Lu}$, J., J. Yu, and C. M. Corvalan (2015). Universal scaling law for the collapse of viscous nanopores. Langmuir 31 (31), 8618-8622.

First theoretical and numerical demonstration of an universal scaling law during the contraction of viscous pores at the sub- 
micron scale, and its relationship with the rheological properties of the host material.

Mason, T., H. Gang, and D. Weitz (1996). Rheology of complex fluids measured by dynamic light scattering. Journal of Molecular Structure 383(1), $81-90$.

Mason, T., H. Gang, and D. Weitz (1997). Diffusing-wave-spectroscopy measurements of viscoelasticity of complex fluids. JOSA A 14 (1), 139-149.

Mason, T. G. and D. Weitz (1995). Optical measurements of frequencydependent linear viscoelastic moduli of complex fluids. Physical review letters $74(7), 1250$.

McGraw, J. D., N. M. Jago, and K. Dalnoki-Veress (2011). Capillary levelling as a probe of thin film polymer rheology. Soft Matter 7(17), 7832-7838.

Mendoza, A. J., E. Guzmán, F. Martínez-Pedrero, H. Ritacco, R. G. Rubio, F. Ortega, V. M. Starov, and R. Miller (2014). Particle laden fluid interfaces: dynamics and interfacial rheology. Advances in colloid and interface science 206, 303-319.

Meyer, A., A. Marshall, B. G. Bush, and E. M. Furst (2006). Laser tweezer microrheology of a colloidal suspension. Journal of Rheology (1978present) 50(1), 77-92.

Moschakis, T. (2013). Microrheology and particle tracking in food gels and emulsions. Current Opinion in Colloid \& Interface Science 18(4), 311-323. 
Moschakis, T., A. Lazaridou, and C. G. Biliaderis (2012). Using particle tracking to probe the local dynamics of barley $\beta$-glucan solutions upon gelation. Journal of colloid and interface science 375 (1), 50-59.

Moschakis, T., B. S. Murray, and E. Dickinson (2010). On the kinetics of acid sodium caseinate gelation using particle tracking to probe the microrheology. Journal of colloid and interface science 345(2), 278-285.

Narita, T. and T. Indei (2016). Microrheological study of physical gelation in living polymeric networks. Macromolecules.

Oelschlaeger, C., M. Cota Pinto Coelho, and N. Willenbacher (2013). Chain flexibility and dynamics of polysaccharide hyaluronan in entangled solutions: a high frequency rheology and diffusing wave spectroscopy study. Biomacromolecules 14(10), 3689-3696.

Peng, X. and G. B. McKenna (2016). Physical aging and structural recovery in a colloidal glass subjected to volume-fraction jump conditions. Physical Review E 93(4), 042603.

**Puertas, A. M. and T. Voigtmann (2014). Microrheology of colloidal systems. Journal of Physics: Condensed Matter 26(24), 243101.

This work offers a comprehensive review of theoretical models available for both passive and active microrheology, with the focus on the application of single-probe motion microrheology in low- and high-density colloidal media.

Rahmani, Y., R. Koopman, D. Denisov, and P. Schall (2014). Visualizing 
the strain evolution during the indentation of colloidal glasses. Physical Review E 89(1), 012304.

Rich, J. P., J. Lammerding, G. H. McKinley, and P. S. Doyle (2011). Nonlinear microrheology of an aging, yield stress fluid using magnetic tweezers. Soft Matter 7(21), 9933-9943.

Rogers, S., C. Van Der Walle, and T. Waigh (2008). Microrheology of bacterial biofilms in vitro: Staphylococcus aureus and pseudomonas aeruginosa. Langmuir 24(23), 13549-13555.

Roth, C. B. and J. R. Dutcher (2006). Hole growth as a microrheological probe to measure the viscosity of polymers confined to thin films. Journal of Polymer Science Part B: Polymer Physics 44 (20), 3011-3021.

Schultz, K. M. and E. M. Furst (2012). Microrheology of biomaterial hydrogelators. Soft Matter 8(23), 6198-6205.

Shayegan, M., T. Altindal, and N. R. Forde (2014). Investigating the mechanism of collagen self-assembly with microrheology. Biophysical Journal 106(2), 55a.

Shayegan, M. and N. R. Forde (2013). Microrheological characterization of collagen systems: from molecular solutions to fibrillar gels. PloS one 8(8), e70590.

*Shindel, M. M. and E. M. Furst (2015). Frequency modulated microrheology. Lab on a Chip 15(11), 2460-2466. 
This work provides an overview of the theory and experimental implementations of Frequency Modulated Microrheology, which enables characterization of the dynamic stress response of complex fluids over several frequency decades in a single experiment.

**Squires, T. M. and T. G. Mason (2010). Fluid mechanics of microrheology. Annual Review of Fluid Mechanics 42(1), 413.

This work reviews the development and current state of various microrheological techniques, and their theoretical justification based on the generalized Stokes-Einstein relation, discussing their range of validity and key assumptions invoked.

Sriram, I., A. Meyer, and E. M. Furst (2010). Active microrheology of a colloidal suspension in the direct collision limit. Physics of Fluids (1994present) 22(6), 062003.

Vadas, E., H. Goldsmith, and S. G. Mason (1973). The microrheology of colloidal dispersions: I. the microtube technique. Journal of Colloid and Interface Science 43(3), 630-648.

Vincent, R., D. Pinder, Y. Hemar, and M. Williams (2007). Microrheological studies reveal semiflexible networks in gels of a ubiquitous cell wall polysaccharide. Physical Review E 76(3), 031909.

Voigtmann, T. and M. Fuchs (2013). Force-driven micro-rheology. The European Physical Journal Special Topics 222(11), 2819-2833.

Willenbacher, N. and C. Oelschlaeger (2007). Dynamics and structure of com- 
plex fluids from high frequency mechanical and optical rheometry. Current Opinion in Colloid 6 Interface Science 12(1), 43-49.

Wilson, L. G. and W. C. Poon (2011). Small-world rheology: an introduction to probe-based active microrheology. Physical Chemistry Chemical Physics 13(22), 10617-10630.

Wirtz, D. (2009). Particle-tracking microrheology of living cells: principles and applications. Annual review of biophysics 38, 301-326.

Xu, J., T. Chang, G. E. Inglett, S. Kim, Y. Tseng, and D. Wirtz (2007). Micro-heterogeneity and micro-rheological properties of high-viscosity oat $\beta$-glucan solutions. Food chemistry 103(4), 1192-1198.

Xu, J., Y. Tseng, C. J. Carriere, and D. Wirtz (2002). Microheterogeneity and microrheology of wheat gliadin suspensions studied by multipleparticle tracking. Biomacromolecules 3(1), 92-99.

Zia, R. N. and J. F. Brady (2010). Single-particle motion in colloids: forceinduced diffusion. Journal of Fluid Mechanics 658, 188-210.

Zia, R. N. and J. F. Brady (2013). Stress development, relaxation, and memory in colloidal dispersions: Transient nonlinear microrheology. Journal of Rheology (1978-present) 57(2), 457-492.

Zia, R. N., B. J. Landrum, and W. B. Russel (2014). A micro-mechanical study of coarsening and rheology of colloidal gels: Cage building, cage hopping, and smoluchowski's ratchet. Journal of Rheology (1978present) 58(5), 1121-1157. 


\section{Figures}

a

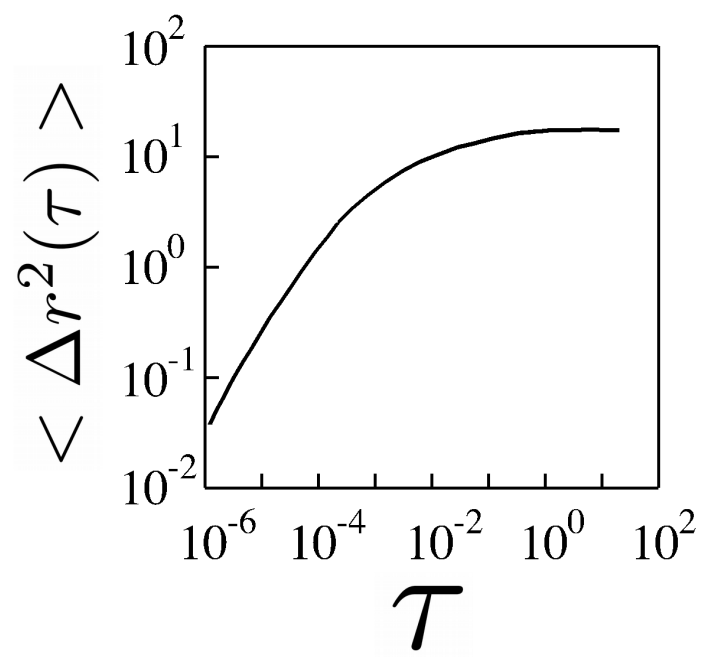

b

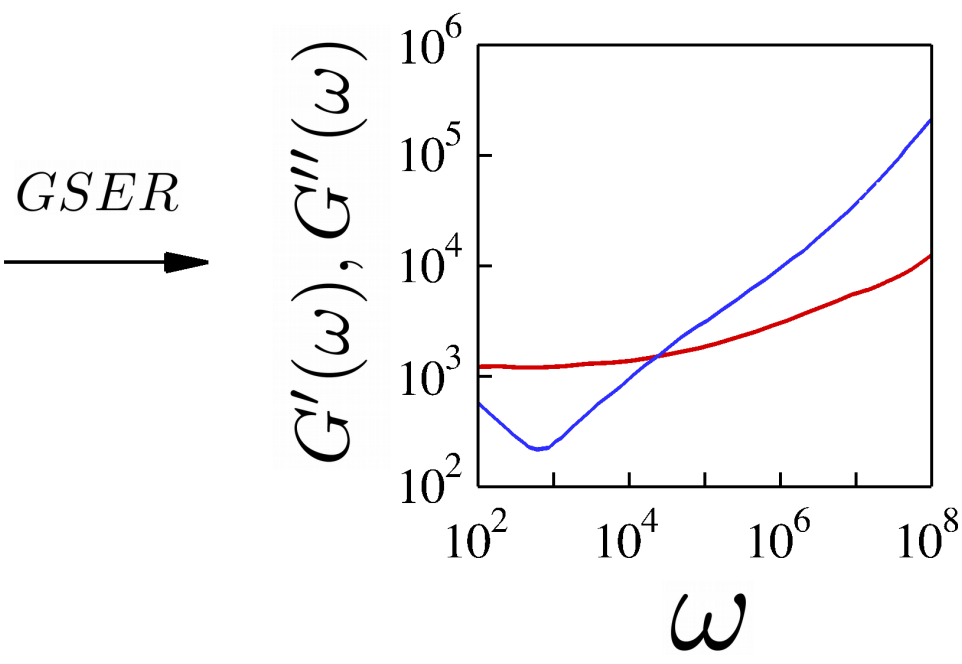

Figure 1: Passive microrheology. Passive microrheology uses the generalized StokesEinstein relation (GSER) and the fluctuation-dissipation theorem to relate the Brownian mean-square displacement $\left\langle\Delta r^{2}\right\rangle$ of a fluctuating probe particle embedded in a soft material as a function of time $\tau$ (a) to the storage modulus $G^{\prime}(\omega)$ (red) and loss modulus $G^{\prime \prime}(\omega)$ (blue) of the material (b). 

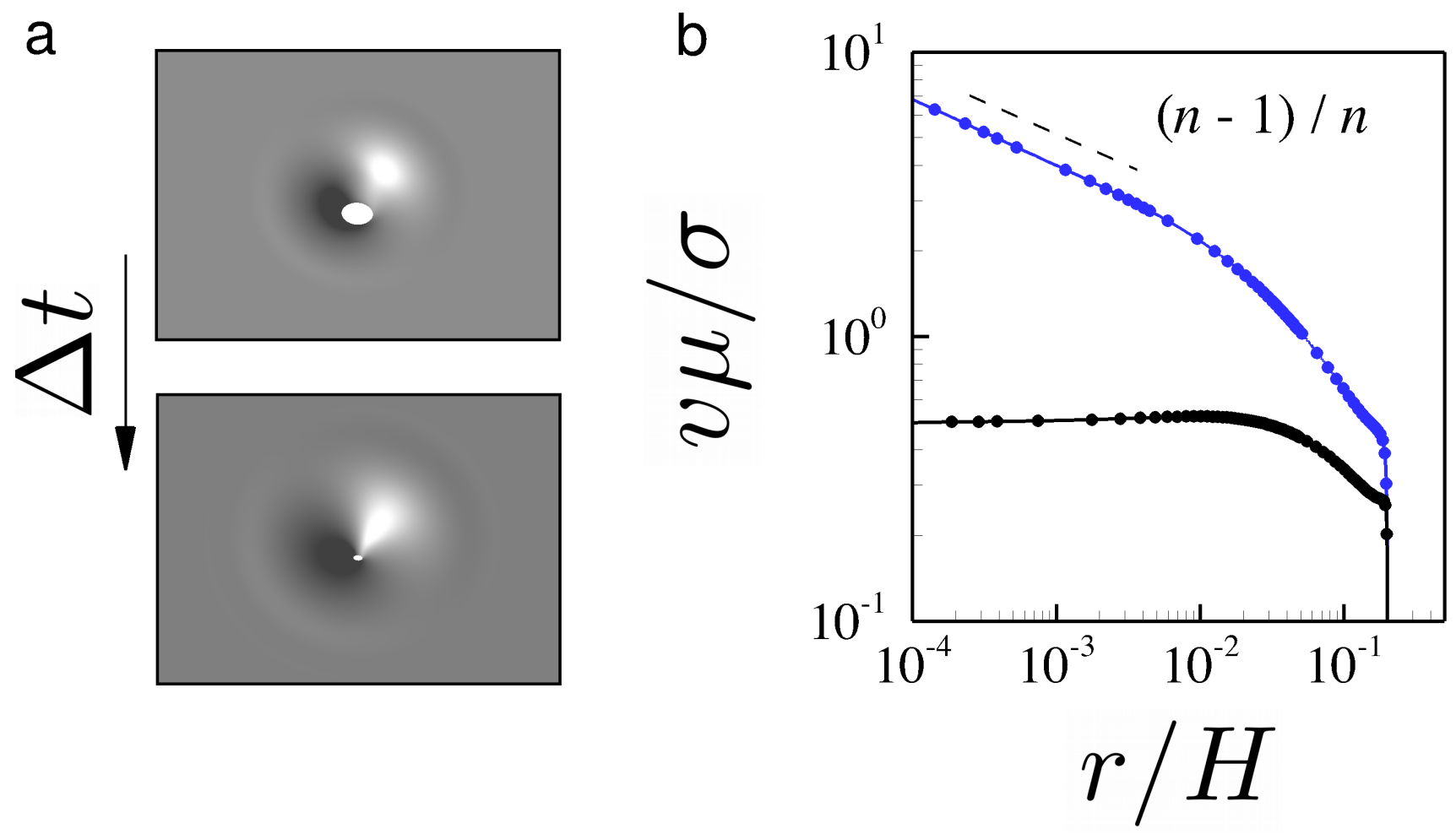

Figure 2: Combined microrheology. Example of a combined technique that relates the capillary velocity $v \mu / \sigma$ of a embedded contracting micropore used as a microrheological probe (a) to the shear-thinning rheological behavior of a soft material (blue line) in which the shear stress decreases $(n<1)$ with increasing shear rate (b). Here, $\Delta t$ is the elapsed time, $n$ the flow index of the material, $r$ the radius of the micropore, and $H$ the thickness of the fluid film. 